\title{
Role of Ultrasound in Assessment of Malignant Risk Potentialof Thyroid Nodule Using TIRADS with Cytological Correlation.
}

\author{
Dr Thaiyalnayakis ${ }^{1}$, DrMurali Nanjundan ${ }^{2}$, Dr Sundari Natarajan ${ }^{3}$, \\ 1,2,3 (Department Of Radio-Diagnosis, Coimbatore Medical College,Tamilnadu, India)
}

\begin{abstract}
:
Background And Objectives: Nearly twothird of the population show nodules in high resolution ultrasound of thyroid. Less than 10\% of nodules are malignant. The reporting of such nodules is often subjective and does not guide proper management. To standardize the reporting, the concept of thyroid imaging reporting and data system (TIRADS) was developed. The present study aims to stratify the thyroid nodules based on key ultrasound features into various risk groups and assess their malignant potential.

Methodology:This study has been done on 200 patients with thyroid nodules during a one year study period(2014-2015). These thyroid nodules were evaluated by ultrasonography and FNAC.Then the sonographic features and FNAC results were compared andanalysed.

Results: Out of the 200 thyroid nodules, 140 nodules were included in TIRADS category 3. TIRADS category $4 a, 4 b$ and 5 had 12, 40, 8 thyroid nodules respectively. All category 3 nodules were proved as benign on FNAC. Malignant nodules were confirmed with increasing frequency from category 4 a to 5 , with $100 \%$ concordance for TIRADS category 5. Odds ratio for irregular margin,microlobulated margin, taller-than-wider shape, microcalcification, marked hypoechogenicity is 1.71, 24, 19.4, 20.35 and 47 respectively.

Conclusion:The sonographic features like irregular margin, microlobulated margin, taller-than-wider shape, microcalcification and marked hypoechogenicity carries increased risk of thyroid malignancy and fine needle aspiration cytology is required only for the nodules which are included in TIRADS category $4 a$, $4 b$ and 5.

Keywords: Fine needle aspiration cytology, TIRADS, thyroid nodules, ultrasonography,

\section{Introduction}

Thyroid gland can be affected by various disease processes which can present clinically as thyroid nodule. Currently, ultrasonography is one of the investigating procedures used for the evaluation of thyroid nodules. But it is not confirmatory to differentiate the benign and malignant thyroid nodules. The investigating procedure which is used for the differentiation of benign from malignant nodule is fine needle aspiration cytology. But it is an invasive and painful procedure. It is also difficult to decide which thyroid nodule needs Fine Needle Aspiration Cytology as current ultrasound reporting of thyroid nodules is often descriptive and subjective. Based on this problem the present study has been undertaken to classify the thyroid nodules into TIRADS (Thyroid Imaging Reporting And Data System) category based on suspicious ultrasound features so as to improve the sensitivity and specificity of ultrasound to differentiate the benign thyroid nodules from malignant thyroid nodules. This may potentially help to restrict FNAC evaluation to nodules showing suspicious imaging features.
\end{abstract}

\section{Objectives}

The objectives of the present study is to classify the thyroid nodules into Thyroid Imaging Reporting And Data System (TIRADS) category based on suspicious ultrasound features and to compare the suspicious ultrasound features with pathological findings of the thyroid nodules to analyse the risk of malignancy .

\section{Methodology}

This study has been undertaken in 200 patients with thyroid nodules admitted in Coimbatore Medical College and Hospital, Coimbatore during the study period of 2014-2015. These patients with thyroid nodules were analysed by their primary thyroid related complaints, the relevant positive and negative histories and proper clinical examination of thyroid gland. The patients were then evaluated by high resolution ultrasonography of thyroid gland for various sonographic findings. The key ultrasound features that were analysed included the shape of the nodules, echogenicity, content, calcification, margins. Based on the ultrasound features the thyroid nodules were stratified into appropriate Thyroid Imaging Reporting And Data System category.

After ultrasound evaluation, these patients underwent ultrasound guided fine needle aspiration cytology. In case of multiple nodules the nodules which had most suspicious sonographic findings for malignancy or the dominant thyroid nodule were selected for fine needle aspiration. Then these FNAC reports 
were compared with ultrasound findings (TIRADS category) of the corresponding nodules and analysed for the association of these ultrasound findings with the benign and malignant thyroid tumors. The ultrasound findings were correlated with histopathological reports of the specimen of thyroid surgery if FNAC reports were indeterminate.

TIRADS 1: Normal

\section{TIRADS Classification}

TIRADS 2:Benign Eg; simple cyst, isolated macrocalcification, thyroiditis.

TIRADS 3: probably benign.

-No high suspicious features.

-Iso echogenic and hyper echogenic nodules

TIRADS 4: probably malignant.

4A:No high suspicious features,

-Moderatelyhypoechogenic nodules

4B: One or two high suspicious features.

-No cervical lymphadenopathy.

TIRADS 5: Malignant

$\geq$ Threehigh suspicious features

\pm lymphadenopathy

\section{Observation}

Ultrasonographically the thyroid nodules were evaluated for shape, echogenicity, content of the nodules, calcification, and margins. The results are shown below.

4.1 Shape: Out of 200 nodules evaluated, 194 nodules were wider than taller in shape and 6 nodules were taller than wider in shape. Among the 194 nodules which were wider than taller in shape, 184 nodules showed benignity on FNAC report. The remaining 10 nodules were malignant in nature on FNAC. All the nodules which showed taller than wider in shape showed malignant character.

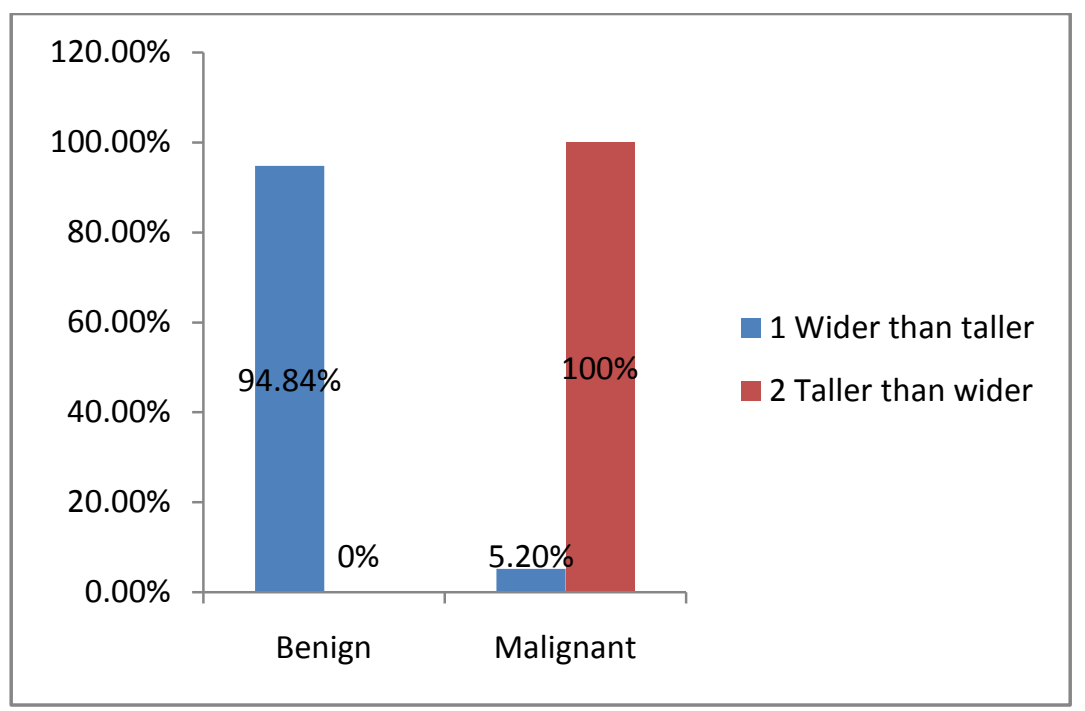

Figure: 1. Chart showing the percentage of thyroid nodules depending upon the Shape

4.2 Echogenicity:Ultrasonographically 172 thyroid nodules showed either isoechogenicity or hyperechogenicity, 16 nodules showed hypoechogenicity and the remaining 12 nodules demonstrated marked hypoechogenicity. All the 172 nodules which had iso or hyperechogenicity on USG have demonstrated benignity on FNAC. Among the 16 nodules which had hypoechogenicity 12 nodules were benign and 4 were malignant on FNAC. All the nodules which showed marked hypoechogenicity were proved malignant on FNAC. 


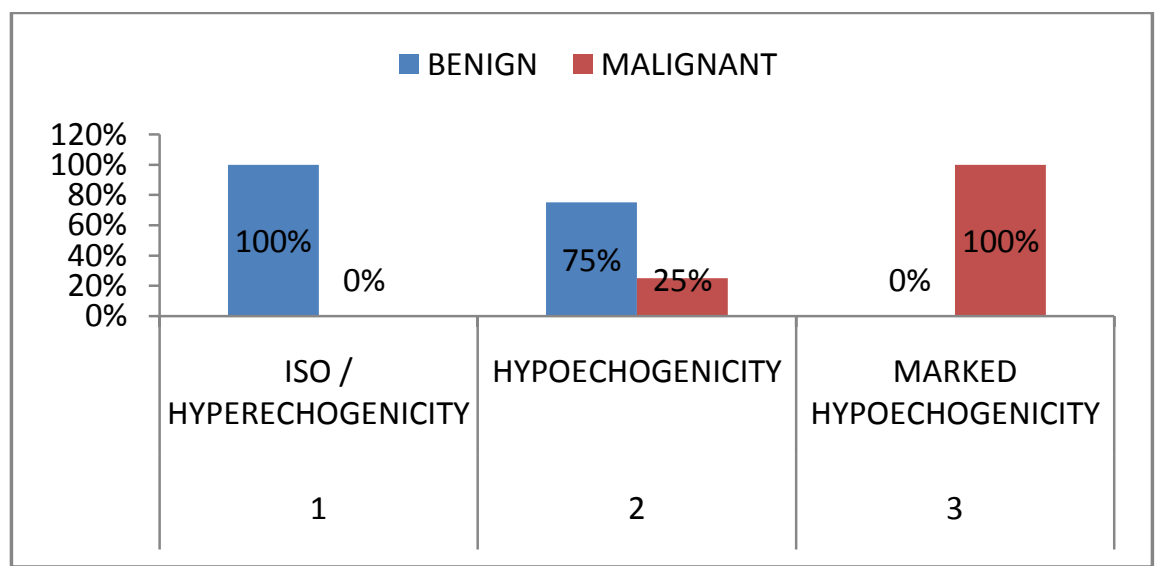

Figure: 2. Chart showing the percentage of thyroid nodules depending upon Echogenicity

4.3 Contents:Ultrasonographically 102 nodules were solid in nature and 98 nodules demonstrated mixed content (solid and cystic). Out of 102 nodules which were solid in nature, 86 nodules were benign and 16 nodules were malignant on FNAC. All 98 nodules with mixed content were benign on FNAC.

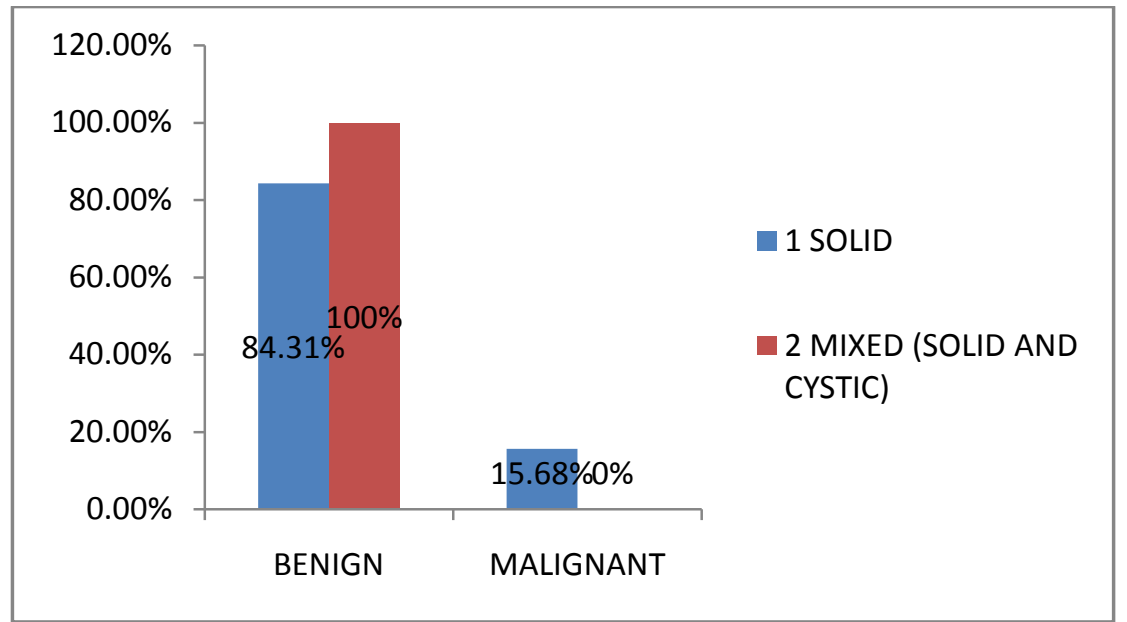

Figure: 3. Chart showing the percentage of thyroid nodules depending upon the Content

4.4 Calcifications: Out of the 200 nodules analysed 176 nodules had no calcification, 10 hadmacrocalcification and 14 had microcalcification. Out of 176 nodules which had no calcification 172 nodules were benign and 4 nodules were malignant on FNAC. All the 10 nodules with macro-calcification had benign character on FNAC. Out of 14 nodules which had micro-calcification, 12 nodules were malignant and 2 nodules were benign on FNAC.

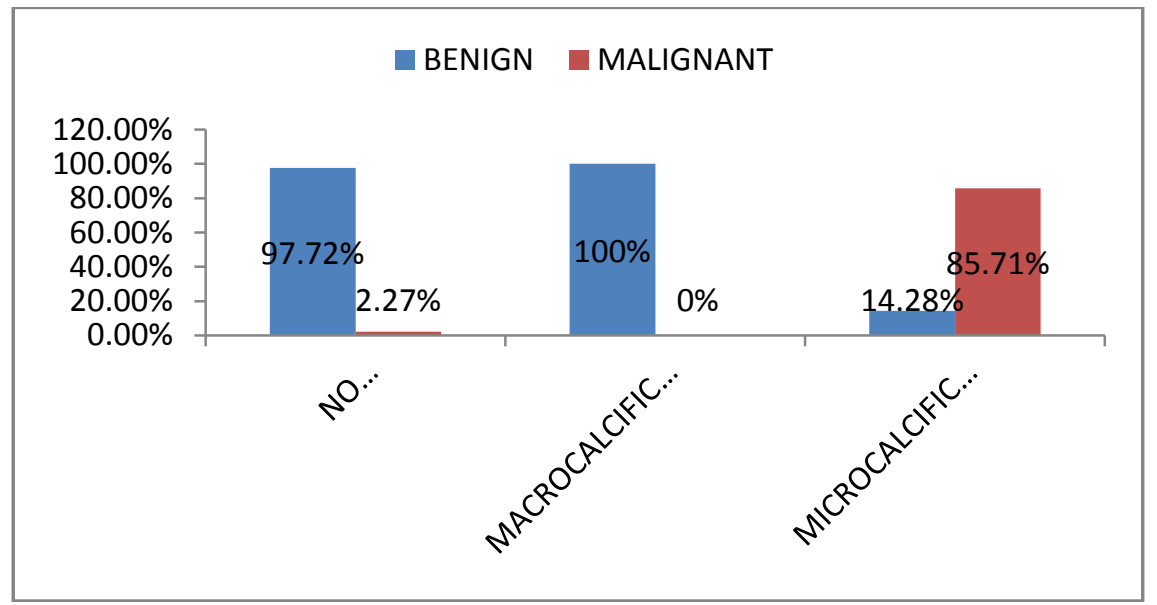

Figure: 4. Chart showing the percentage of thyroid nodules depending upon the Calcification 
4.5 Margins:Among the 200 nodules, 140 nodules had smooth margin, 52 nodules had irregular margin and 8 nodules had microlobulated margin on USG. Out of 140 nodules which had smooth margin on USG, 138 nodules were benign and 2 nodules were malignant on FNAC. Among the 52 nodules which had irregular margin 46 were benign and 6 were malignant on FNAC. Out of 8 nodules which had microlobulated margin, all were proved malignant on FNAC.

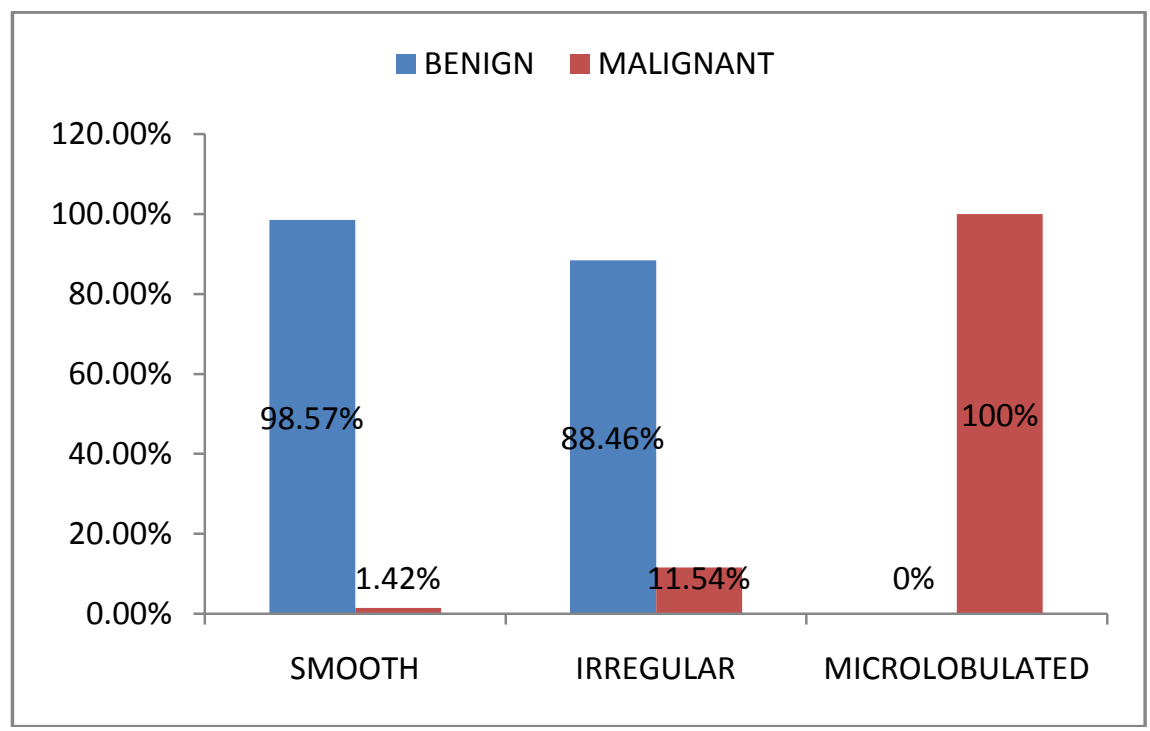

Figure: 5. Chart showing the percentage of thyroid nodules depending upon the margin

According to the ultrasound features all the thyroid nodules were categorized into TIRADS category. Out of the 200 nodules evaluated 140 nodules were included in TIRADS category 3, 12 nodules in category 4a and 40 nodules in category $4 \mathrm{~b}$. The remaining 8 nodules were included under category 5 . All the nodules in category 3 were shown benign character on histopathology. Two nodules in category $4 \mathrm{a}$ and six nodules in category $4 \mathrm{~b}$ were malignant and all the 8 nodules in category 5 had malignant character in histopathology.

Table: 1. TIRADS category \&FNAC result

\begin{tabular}{|c|c|c|c|}
\hline \multicolumn{2}{|c|}{ USG Stratification } & \multicolumn{2}{c|}{ FNAC Result } \\
\hline Category & No Of Nodules & Benign & Malignant \\
\hline TIRADS 3 & 140 & 140 & 0 \\
\hline TIRADS 4a & 12 & 10 & 2 \\
\hline TIRADS 4b & 40 & 34 & 6 \\
\hline TIRADS 5 & 8 & 0 & 8 \\
\hline
\end{tabular}

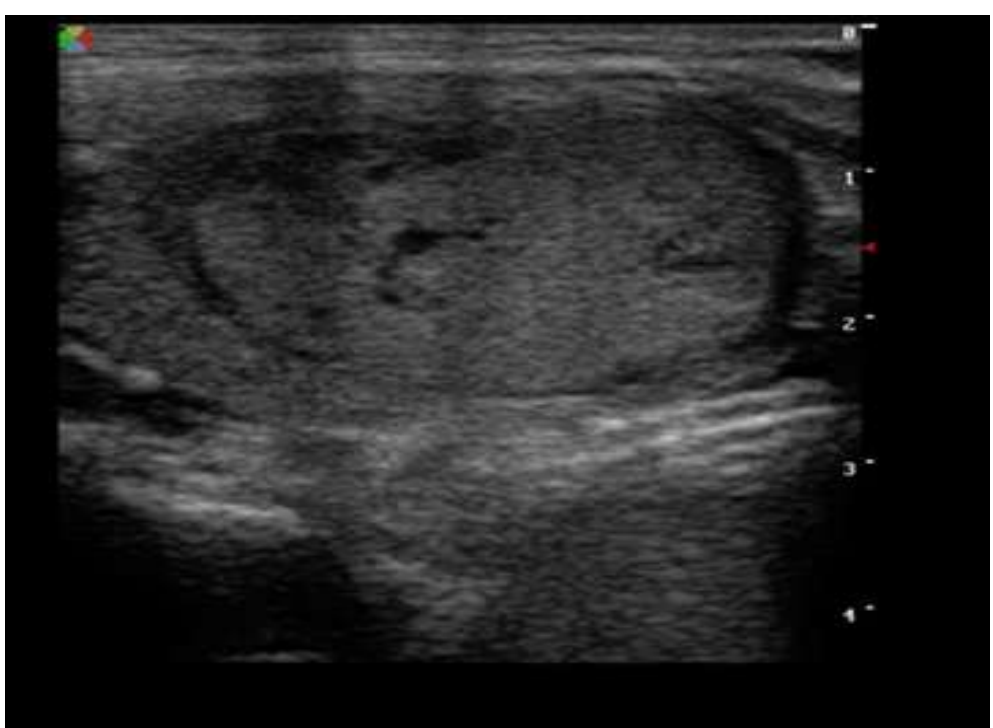

Figure: 6Picture showing features of benign nodule (TIRADS 2)-oval shape,isoehogenicity, smooth margin, solid and cystic content. 


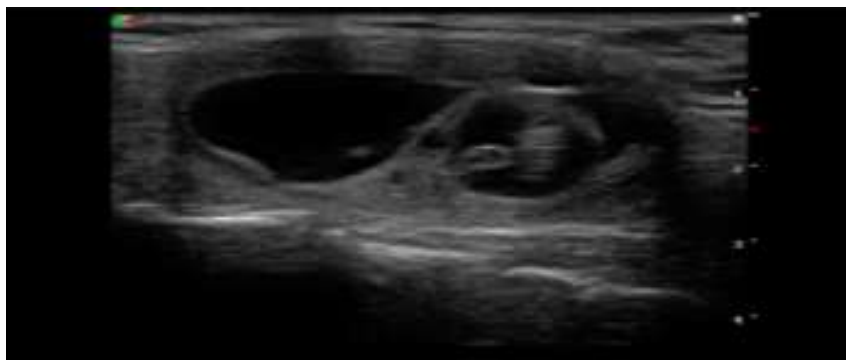

Figure: 7Image showing thyroid nodule with wider than taller shape, isoechogenicity, solid with cystic changes, smooth margin. (TIRADS category 3) FNAC showed nodular goiter with cystic degeneration)

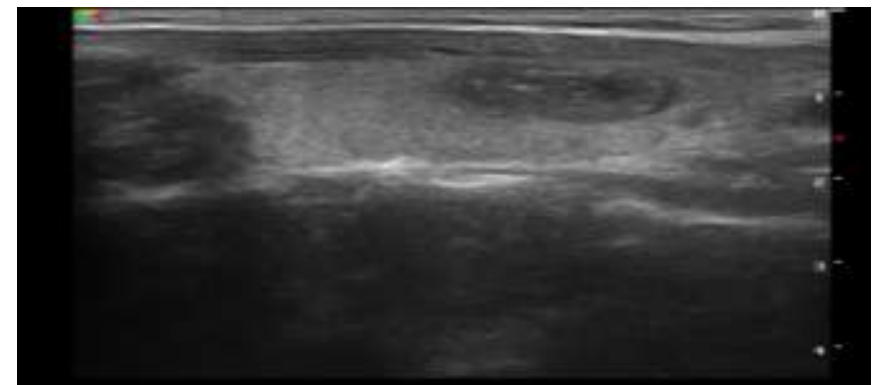

Figure: 8Image showing thyroid nodule with marked hypoechogenicity, solid content, smooth margin, wider than taller shape. (TIRADS category 4b).FNAC showed follicular thyroid carcinoma.

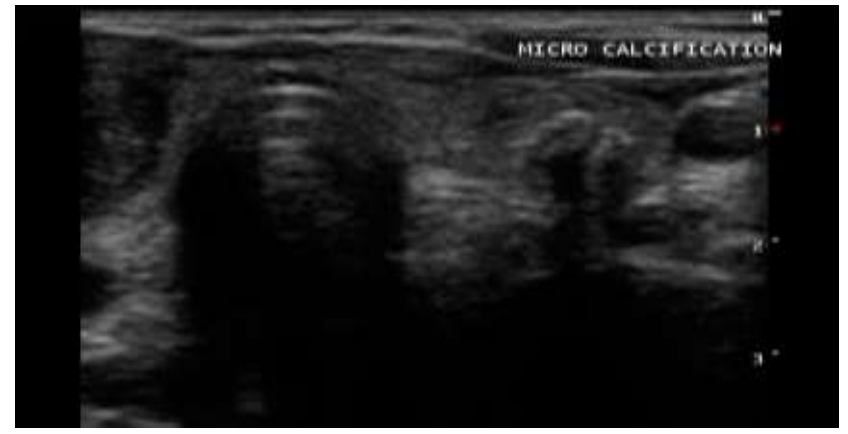

Figure:9Image showing thyroid nodule with taller than wider shape, marked hypoechogenicity, solid content, irregular margin, microcalcification(TIRADS category 5). FNAC showed papillary thyroid carcinoma

\section{Discussion}

Nearly two third of the population show nodules in high resolution ultrasound of thyroid. [1,2]. In some studies it was stated that less than $10 \%$ of thyroid nodules are malignant. In the present study $8 \%$ of thyroid nodules were malignant on histopathology. It correlates with the previous studies.

In a study conducted by Moifo B et al it was stated that the risk of malignancy increased from TIRADS category 2 to category 5 from $2.2 \%$ to $100 \%$ respectively. Risk of malignancy inour study is compared with study by Moifo et al in the following table.

Table: 2. Comparison of TIRADS category between two studies

\begin{tabular}{|c|c|c|c|}
\hline S. No & $\begin{array}{c}\text { TIRADS } \\
\text { category }\end{array}$ & $\begin{array}{c}\text { Risk of malignancy in study } \\
\text { by Moifo et al. }\end{array}$ & $\begin{array}{c}\text { Risk of malignancy in } \\
\text { present study }\end{array}$ \\
\hline 1. & Category 3 & $2.2 \%$ & $0 \%$ \\
\hline 2. & Category 4a & $5.9 \%$ & $16.7 \%$ \\
\hline 3. & Category 4b & $57.9 \%$ & $15 \%$ \\
\hline 4. & Category 5 & $100 \%$ & $100 \%$ \\
\hline
\end{tabular}

Horvath et al suggested that the risk of malignancy for TIRADS category 3, 4a, 4b was less than 5\%, 5 $-10 \%, 10-80 \%$ respectively. The TIRADS category 5 carries greater than $80 \%$ risk of malignancy [3]. Our results are corresponding to the previous study done by Horvath et al, except for TIRADS category 4a in which we had slightly increased risk for malignancy. This can be explained by the fact that the assessment of the margins of the thyroid nodules was subject to inter observer variation. 
Moifo et al reported that the presence of irregular contour of the thyroid nodules had sensitivity and specificity of $34.78 \%$ and $99.51 \%$ respectively. The positive predictive value and negative predictive value are also very high.Odds ratio is 22.40 (CI - 12.47 - 40.23). In our present study, the presence of irregular margin had sensitivity, specificity, positive predictive value and negative predictive value of $37.5 \%, 75 \%, 11.54 \%$ and $93.24 \%$ respectively with the odds ratio $1.71(\mathrm{CI}-0.65-4.47)$.

Table: 3. Comparison of two studies for irregular margin

\begin{tabular}{|c|c|c|c|c|c|c|}
\hline S.No & Study & Sensitivity (\%) & $\begin{array}{c}\text { Specificity } \\
(\%)\end{array}$ & PPV (\%) & NPV (\%) & OR \\
\hline 1. & Moifo B et al & 34.78 & 99.51 & 80 & 96.43 & 22.40 \\
\hline 2. & Present study & 37.5 & 75 & 11.54 & 93.24 & 1.71 \\
\hline
\end{tabular}

In our present study, the sensitivity, specificity, positive predictive value and negative predictive value for the microlobulated margin are 50\%, 100\%, $100 \%$ and $95.83 \%$ respectively. Odds ratio for the microlobulated margin is 24 (CI - 12.18-47.30).

Moifo B et al reported that the thyroid nodules with taller-than-wider shape had sensitivity, specificity, positive predictive value and negative predictive value of $4.35 \%, 100 \%, 100 \%$ and $94.81 \%$ respectively with odds ratio of 19.50 . In our present study taller-than-wider shape had sensitivity of $37.5 \%$, negative predictive value of $94.84 \%$ and $100 \%$ specificity and positive predictive value. The odds ratio for taller-than-wider nodule is $19.4(\mathrm{CI}-10.61-35.48)$.

Table: 4. Comparison of two studies for taller-than-wider shape

\begin{tabular}{|c|c|c|c|c|c|c|}
\hline S.No & Study & Sensitivity (\%) & Specificity (\%) & PPV (\%) & NPV (\%) & OR \\
\hline 1. & Moifo B et al & 4.35 & 100 & 100 & 94.87 & 19.50 \\
\hline 2. & Present study & 37.5 & 100 & 100 & 94.84 & 19.40 \\
\hline
\end{tabular}

In our current study the presence of microcalcification had sensitivity, specificity, positive predictive value and negative predictive value of $75 \%, 98.91 \%, 85.71 \%$ and $97.85 \%$ respectively. It also had the odds ratio 20.35 (CI - 10.00 - 41.45). In the study by Moifo B et al, the odds ratio for microcalcification is 15.24 (CI 7.74 - 30.02). So according to both the studies, the presence of microcalcification carries higher risk of malignancy than the nodules without microcalcification[4].

Table: 5. Comparison of two studies for micro-calcification

\begin{tabular}{|c|c|c|c|c|c|c|}
\hline S.No & Study & Sensitivity (\%) & $\begin{array}{c}\text { Specificity } \\
(\%)\end{array}$ & PPV (\%) & NPV (\%) & OR \\
\hline 1. & Moifo B et al & 30.4 & 98.8 & 58.3 & 96.2 & 15.24 \\
\hline 2. & Present study & 75 & 100 & 100 & 97.87 & 20.35 \\
\hline
\end{tabular}

In our present study, presence of marked hypoechogenicity had sensitivity, specificity, positive predictive value and negative predictive value of $75 \%, 100 \%, 100 \%$ and $97.87 \%$ respectively with the odds ratio 47 ( CI - 17.83 - 123.92). So the thyroid nodule with marked hypoechogenicity has 47 times increased risk of malignancy than the nodule without marked hypoechogenicity.

Table:6. Comparison of two studies for marked hypoechogenicity

\begin{tabular}{|c|c|c|c|c|c|c|}
\hline S.No & Study & Sensitivity (\%) & Specificity (\%) & PPV (\%) & NPV $(\%)$ & OR \\
\hline 1. & Moifo B et al & 13.04 & 99.51 & 60 & 95.29 & 12.75 \\
\hline 2. & Present study & 75 & 100 & 100 & 97.87 & 47 \\
\hline
\end{tabular}

In another study done by Kwak et al, the odds ratio for marked hypoechogenicity, microlobulated margins, irregular margins, microcalcification and taller-than-wider shape was 69.756 (CI - 37.216 - 130.747), 20.135 (CI - 14.038 - 28.880), 113.828 (CI - 60.771 - 213.205), 25.871 (CI - 17.503 - 38.240) and 24.478 (CI $-17.152-34.933)^{5}$. According to this study all the above mentioned sonographic features are associated with increased risk of malignancy.

Table: 7. Comparison of odds ratio for suspicious ultrasound features

\begin{tabular}{|c|c|c|c|c|c|}
\hline & $\begin{array}{c}\text { Marked } \\
\text { Hypoechogenicity } \\
(\mathrm{MH})\end{array}$ & $\begin{array}{c}\text { Microlobulated } \\
(\text { ML) Margin }\end{array}$ & $\begin{array}{c}\text { Irregular } \\
\text { (IR) } \\
\text { Margin }\end{array}$ & $\begin{array}{c}\text { Micro } \\
\text { calcification } \\
\text { (MIC CAL) }\end{array}$ & $\begin{array}{c}\text { Taller-than- } \\
\text { wider (TTW) } \\
\text { shape }\end{array}$ \\
\hline $\begin{array}{c}\text { 1.Moifo B et } \\
\text { al }\end{array}$ & 12.75 & - & 22.40 & 15.24 & 19.50 \\
\hline 2.Kwak et al & 69.756 & 20.135 & 113.828 & 25.871 & 24.478 \\
\hline $\begin{array}{c}\text { 3.Present } \\
\text { study }\end{array}$ & 47 & 24 & 1.71 & 20.35 & 19.4 \\
\hline
\end{tabular}


According to the current study, taller-than-wider shape, marked hypoechogenicity and microlobulated margins in ultrasonography are $100 \%$ specific for malignancy. There are certain sonographic features consistently associated with benign nodules. These features include isoechogenicity or hyperechogeicity, mixed (solid and cystic) content of the nodules and the presence of macrocalcification. These sonographic features help to exclude the malignant thyroid nodules. However there are rare exceptions like cystic necrosis of papillary carcinoma which was not encountered in our current study.

\section{Conclusion}

The present study showed that TIRADS category 3 has $0 \%$ risk of malignancy and category 5 has $100 \%$ risk of malignancy. The risk of malignancy increases from TIRADS category 3 to TIRADS category 5. So the thyroid nodules which are included in TIRADS category 3 or less than 3 do not need FNAC. They require short term follow up every 6 months to document stability of the lesion. Only the nodules included in TIRADS category 4 and 5 require biopsy to differentiate the benign from malignant thyroid nodules.

The sonographic features like irregular margins, microlobulated margins, marked hypoechogenicity, microcalcification and taller-than-wider shape are all associated with increased risk of malignancy. Presence of microlobulated margins, marked hypoechogenicity and taller-than-wide shaped nodules are $100 \%$ specific for malignancy. Sonographic features like isoechogenicity or hyperechogenicity, mixed (solid and cystic) content of the nodule and macrocalcification are predictive of benignity.

Using TIRADS system in routine reporting of thyroid nodules improves objectivity of reports, gives fairly accurate assessment of malignant risk potential, gives clear information regarding further course of action (no action required or observation or FNAC) and therebyavoidsunnecessary FNAC of obviously benign category 2 or 3 lesions.

[1]. Kim K M, Park J B et al. Ultrasonographic guideline for thyroid nodules cytology: Single institute experience, Journal of the Korean surgical society, 2013; vol 84 (2): 73-9.

[2]. Dean D S et al. Epidemiology of thyroid nodules, Best practice and Research: clinical endocrinology and metabolism, Journal of radiology, 2012; vol 262(3): 901-11.

[3]. Horvath E, Majlis S et al. An ultrasonogram reporting system for thyroid nodules stratifying cancer risk for clinical management, The journal of clinical endocrinology and metabolism, 2009; vol 9 (5): 1748-51.

[4]. Moifo B, Takoeta et al. Reliability of Thyroid Imaging Reporting and Data System (TIRADS) classification in Differentiation Benign from Malignant Thyroid Nodules, Open Journal of Radiology, 2013; 3: 103 - 7 .

[5]. Kawk Y J, Han H K et al. Thyroid Imaging Reporting and Data System for US features of Nodules: A step in establishing better stratification of cancer risk, RSNA, 2011; vol 260: 892-99.

[6]. Rumack C M, Wilson S R et al. Diagnostic Ultrasound, Volume 1, $4^{\text {th }}$ edn, Chapter 18; The thyroid gland, ELSEVIER: Chaina, $2011 ; 708-46$

[7]. Kwak J Y, Jung I et al. Image Reporting and characterization system for ultrasound features of Thyroid nodules: Multicentric Korean Retrospective Study, Korean J Radiol, 2013; 14(1):110 -17. 